\title{
CORRECTION
}

Check for updates

Cite this: New J. Chem., 2019, 43, 12511

DOI: $10.1039 / c 9 n j 90102 g$

rsc.li/njc

\section{Correction: PCL-DOX microdroplets: an evaluation of the enhanced intracellular delivery of doxorubicin in metastatic cancer cells via in silico and in vitro approaches}

\author{
Aman Chandra Kaushik, ${ }^{\text {ah }}$ Ajay Kumar, ${ }^{\text {bc }}$ Chun-Yen Yu, ${ }^{d}$ Shiao-Wei Kuo, ${ }^{e}$ \\ Shih-Shin Liang, ${ }^{f}$ Satya P. Singh, ${ }^{9}$ Xiangeng Wang, ${ }^{a}$ Yan-Jing Wang, ${ }^{a}$ \\ Chung-Kun Yen, ${ }^{\mathrm{c}}$ Xiaofeng Dai, ${ }^{\mathrm{h}}$ Dong-Qing Wei, ${ }^{* a}$ Cheng-Tang Pan ${ }^{* \mathrm{ci}}$ and \\ Yow-Ling Shiue* ${ }^{\star b}$
}

Correction for 'PCL-DOX microdroplets: an evaluation of the enhanced intracellular delivery of doxorubicin in metastatic cancer cells via in silico and in vitro approaches' by Aman Chandra Kaushik et al., New J. Chem., 2019, DOI: 10.1039/c9nj01902b.

The authors would like to correct the Acknowledgements section, as the funding information is incorrect in the published article. The Acknowledgements section should read:

This work was financially supported by Grant CMRPG8G0361 from the Chang Gung Memorial Hospital, R.O.C., and the Ministry of Science \& Technology, Taiwan, under contract MOST 107-2221-E-110-031-MY3 and 107-2221-E-110-032-MY3.

The Royal Society of Chemistry apologises for these errors and any consequent inconvenience to authors and readers.

\footnotetext{
${ }^{a}$ The State Key Laboratory of Microbial Metabolism, School of Life Sciences and Biotechnology, Shanghai Jiao Tong University, Shanghai, 200240, China. E-mail: amanbioinfo@sjtu.edu.cn,wangxiangeng@sjtu.edu.cn, tangtangfeiwu@qq.com,dqwei@sjtu.edu.cn

${ }^{b}$ Institute of Biomedical Sciences, National Sun Yat-Sen University, Kaohsiung City 804, Taiwan. E-mail: ajaynsysu@mem.nsysu.edu.tw, shirley@imst.nsysu.edu.tw ${ }^{c}$ Department of Mechanical and Electro-Mechanical Engineering, National Sun Yat-Sen University, Kaohsiung City 804, Taiwan. E-mail: alden0113@gmail.com, pan@mem.nsysu.edu.tw

${ }^{d}$ From the Liver Transplantation Program and Departments of Diagnostic Radiology, Kaohsiung Chang Gung Memorial Hospital and Chang Gung University College of Medicine, Kaohsiung, Taiwan. E-mail: y7192215@ms17.hinet.net

${ }^{e}$ Department of Materials and Optoelectronic Science, National Sun Yat-Sen University, Kaohsiung 80424, Taiwan. E-mail: kuosw@faculty.nsysu.edu.tw

${ }^{f}$ Department of Biotechnology, College of Life Science, Kaohsiung Medical University, Kaohsiung, Taiwan. E-mail: liang0615@kmu.edu.tw

${ }^{g}$ School of Electrical and Electronic Engineering, Nanyang Technological University, Singapore. E-mail: satya002u@gmail.com

${ }^{h}$ Wuxi School of Medicine, Jiangnan University, Wuxi, China. E-mail: xiaofeng:dai@jiangnan.edu.cn

${ }^{i}$ Institute of Medical Science and Technology, National Sun Yat-Sen University, Kaohsiung City 804, Taiwan
} 